\title{
Prospective comparative study of culture specimens and methods in diagnosing influenza in adults
}

\author{
M L Schmid, G Kudesia, S Wake, R C Read
}

Influenza is diagnosed by culturing influenza virus in respiratory secretions. ${ }^{1}$ Although childhood infection with respiratory syncytial virus is detected by culturing nasopharyngeal aspirates, in adults with influenza throat swabs are generally taken. This report compares culture of nasopharyngeal aspirates with culture of throat swabs and rapid culture methods with standard methods in detecting influenza virus.

\section{Subjects, methods, and results}

Between November 1995 and January 1996, 41 patients were admitted to the Infectious Disease Unit at the Royal Hallamshire Hospital, Sheffield, with symptoms and signs consistent with influenza. Thirty nine of these patients gave their informed consent to undergo nasopharyngeal aspiration in addition to routine investigations. The procedure was performed with a small suction catheter and trap. Viral transport medium was added to the aspirate and samples were sent to the laboratory within 24 hours. Nasopharyngeal aspirates and throat swabs were cultured by standard methods in primary monkey kidney cells at $33^{\circ} \mathrm{C}$ and $37^{\circ} \mathrm{C}$. In addition, rapid culture was performed on nasopharyngeal aspirates, throat swabs, and viral transport medium; samples were centrifuged on coverslips in shell vials and then incubated at $37^{\circ} \mathrm{C}$ before fixation with methanol after 24 hours and 48 hours. The specimens were then stained with fluorescein isothiocyanate. ${ }^{2}$ For direct immunofluorescence, cells from nasopharyngeal aspirates were air dried, fixed on slides coated with Teflon, and stained with the labelled influenza A and B monoclonal antibodies.

Cultures from 17 of the 39 patients were positive for influenza $\mathrm{A}\left(\mathrm{H}_{3} \mathrm{~N}_{2}\right)$. Of the cultures from the 22 patients negative for influenza virus, six yielded an enterovirus, one herpes simplex virus- 1 , and 15 no virus. The table shows the sensitivity of nasopharyngeal aspirates and throat swabs subjected to standard culture and rapid culture in yielding a positive culture. Nasopharyngeal aspirates were twice as sensitive as throat swabs. No patient had virus isolated from a throat swab alone, whereas in nine patients virus was isolated only from nasopharyngeal aspirates. Rapid culture correctly identified 13 of the 17 standard positive nasopharyngeal aspirate samples, but 12 of the 13 rapid cultures became positive within 24 hours compared with 4-20 days with the standard technique. Direct immunofluorescence of the nasopharyngeal aspirate was the least sensitive diag- nostic method, identifying only four of the positive samples $(\mathrm{P}<0.001$ in comparison with standard culture, McNemar's test), with no false positive results.

\section{Comment}

These data were obtained during the height of the influenza A $\left(\mathrm{H}_{3} \mathrm{~N}_{2}\right)$ season between October 1995 and March $1996^{3}$ and showed that rapid culture of nasopharyngeal aspirates is potentially useful for the early diagnosis of influenza virus infection. If a throat swab had been the only sample cultured a positive diagnosis would have been missed in half the patients with influenza A. Rapid culture of nasopharyngeal aspirates was positive in only $76 \%$ of the patients with a positive culture result but does have the advantage over standard culture of yielding a result within 24 hours instead of 1-3 weeks. Gene amplification and new antigen detection techniques are comparatively untested and are not available at most centres. The rapid virus culture technique is already established ${ }^{2}$ and uses technology available in most routine laboratories. The rapid diagnosis of influenza will assume greater importance as new treatments against viruses become available. ${ }^{4}$ Nasopharyngeal aspiration is easily conducted with only minor discomfort to the patient. Adequate amounts of sample can be ensured by washing the aspiration tube with transport medium once the procedure is completed. Other diagnostic techniques routinely used include direct immunofluorescence and serodiagnosis, but these are of little value in acutely ill patients. Our data agree with those of other studies that direct immunofluorescence is less sensitive than culture. ${ }^{4}$

Contributors: GK and RM conceived the study. MLS identified patients and collected samples from them. GK designed the protocol for the laboratory investigation. SW performed the laboratory work. RCR designed the protocol for patient identification and investigation and dealt with ethical issues. RCR, GK, and MLS wrote the paper. RCR is the guarantor.

Funding: This work was supported by the Special Trustees of the Former United Sheffield Hospitals.

Conflict of interest: None.

1 Betts RF. Influenza virus. In: Mandell GL, Bennett, JE, Dolin R, eds. Principles and practice of infectious diseases. New York: Churchill Livingstone, $1995,1546-67$.

2 Schirm J, Luijit DS, Pastoor GW, Mandema JM, Schroder FB. Rapid detection of respiratory viruses using mixtures of monoclonal antibodies on shell vial cultures. Med Virol 1992;38:147-51.

3 Hutchinson EJ, Joseph CA, Zambon M, Fleming DM, Watson JM. Influenza surveillance in England and Wales: October 1995 to June 1996. Commun Dis Rep CDR Wkly 1996;6:163-9.

4 Wiselka M. Influenza: diagnosis, management and prophylaxis. $B M J$ 1994:308:1341-5.

(Accepted 3 June 1997)
North Trent Department of Infection and Tropical Medicine, Royal Hallamshire Hospital, Sheffield S10 2JF

Matthias L Schmid, specialist registrar in infectious diseases

Public Health Laboratory,

Northern General Hospital, Sheffield S5 7AU

Goura Kudesia, consultant virologist

Shaun Wake, medical laboratory scientific officer

Division of

Molecular and

Genetic Medicine, University of

Sheffield Medical

School, Sheffield

S10 2RX

Robert C Read,

senior lecturer in infectious diseases

Correspondence to: Dr Read r.c.read@sheffield. ac.uk

BMJ 1998;316:275

Comparison of results of culture of nasopharyngeal aspirates and throat swabs in 17 patients positive for influenza virus

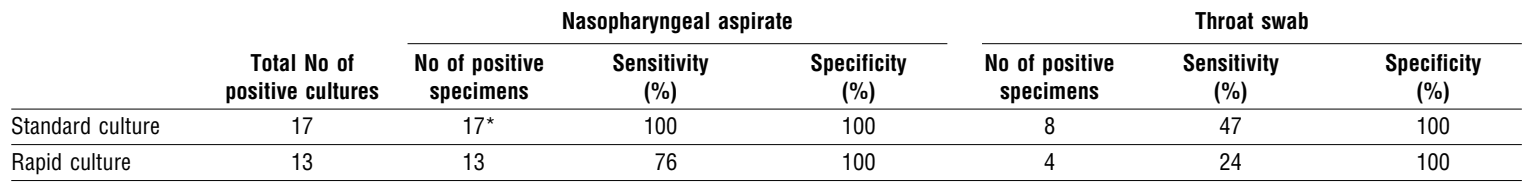

${ }^{*}$ Standard culture of nasopharyngeal aspirates $v$ standard culture of throat swab $(P=0.008$, McNemar's test). Standard culture of nasopharyngeal aspirates $v$ rapid culture of nasopharyngeal aspirate not significant. $\mathrm{P}=0.13$. 